\title{
Simplified Matrix Focusing Imaging Algorithm for Ultrasonic Nondestructive Testing
}

\author{
Xinyu Zhao, Zemin Ma and Jiaying Zhang ${ }^{*}$ (i)
}

\begin{abstract}
Full matrix focusing method of ultrasonic phased array has been proved with advantages of good signal-to-noise ratio and imaging resolution in the field of Ultrasonic NDT. However, it is still suffering from the time-consuming data acquisition and processing. In order to solve the problem, two simplified matrix focusing methods are provided in the paper. One provided method is a triangular matrix focusing algorithm based on the principle of reciprocity for the multi-channel ultrasonic system. The other provided method is a trapezoidal matrix focusing algorithm based on the energy weight of the different channel to the focusing area. Time of data acquisition and computational is decreased with the provided simplified matrix focusing methods. In order to prove the validity of two provided algorithms, both side-drilled holes and oblique cracks are used for imaging experiments. The experimental results show that the imaging quality of the triangular matrix focusing algorithm is basically consistent to that of the full matrix focusing method. And imaging quality of the trapezoidal matrix focusing algorithm is slightly reduced with the amount of multi-channel data decreasing. Both data acquisition and computational efficiency using the triangular matrix focusing algorithm and the trapezoidal matrix focusing algorithm have been improved significantly compared with original full matrix focusing method.
\end{abstract}

Keywords: Ultrasonic NDT, Phased array, Full matrix focusing, Imaging algorithms

\section{Introduction}

In recent years, ultrasonic phased array technology has been developed rapidly [1-4]. And ultrasonic phased array technology has been gradually applied in the field of industrial nondestructive testing [5-8]. The phased array imaging algorithms have been widely studied [9-12]. Holmes et al. [13] proposed the concept of full-matrix focus capture (FMC), and set up the total focus method (TFM) algorithm using FMC. Compared with the conventional ultrasonic phased array testing, the geometric features of defects are more clearly with TFM technology. And the image quality of TFM technology is significantly better than the traditional phased array imaging algorithm with steering and focusing time delays. Wu et al. [14] extended the total focusing imaging method to the

*Correspondence: zjy@djtu.edu.cn

School of Materials Science and Engineering, Dalian Jiaotong University, Dalian 116028, China multi-mode case with both direct and reflection waves. Pan et al. [15] used the total focusing imaging method to simulate the cracks quantitatively. Wu et al. [16] studied primary reflection detection mode based on the optimized direct wave detection, and provided the multimode composite full focus imaging method. Aiming at the problem of internal defect detection of aluminum container weld, Wang et al. [17] proposed an ultrasonic array full focus imaging method based on oblique incidence.

However, TFM imaging is a time-consuming method due to the large amount of full matrix data [18-21], and it is difficult to obtain real-time image [22-25]. In order to improve the efficiency of TFM, Ran [26] used FPGA to analyzing the principle of full focus synthetic aperture imaging algorithm, and designed software to optimize the algorithm for results. Jin et al. [27] introduced the wavenumber algorithm to improving computational efficiency for ultrasonic full-matrix imaging 
in multi- layered medium. Post-processing speed of full matrix data is increased significantly with the provided method. The binary particle swarm optimization (BPSO) algorithm is applied to optimize the array layout. The BPSO algorithm produced favorable results in the case of small-scale sparse array [28]. Hu et al. [29, 30] optimized the locations of active array elements in the sparse array with the genetic algorithm.

Two simplified matrix focusing methods are proposed in the paper. One is the triangular matrix focusing algorithm based on the reciprocity principle of the transmitter and receiver channel, named as triangular matrix focusing method. The other one is the trapezoidal matrix focusing algorithm based on the energy weight of the distance from different channels to the focusing point, named as trapezoidal matrix focusing method.

In Section 2, the data acquisition and imaging algorithm of full matrix focusing method is introduced, and the simplified imaging methods are provided. In Section 3, side-drilled holes and oblique crack defects imaging experiments are carried out. The experimental results are analyzed to compare the imaging quality and computational efficiency with three image algorithms.

\section{Focus Imaging Algorithm}

\subsection{Full Matrix Focused Data Acquisition}

A-scan data is acquired and arranged in a matrix for imaging. Taking a linear array with $\boldsymbol{n}$ elements as an example, the 1st element is fired and all elements are received. The acquired data is named as $A_{11}, A_{12}, \ldots, A_{1 \mathrm{n}}$. And the acquired data is placed in the first row. Then next element is excited in turn until all the $\boldsymbol{n} \times \boldsymbol{n} \mathrm{A}$-scan data are acquired as listed in Eq. (1).

$$
\left[\begin{array}{ccccc}
A_{11} & A_{12} & A_{13} & \cdots & A_{1 n} \\
A_{21} & A_{22} & A_{23} & \cdots & A_{2 n} \\
A_{31} & A_{32} & A_{33} & \cdots & A_{3 n} \\
\vdots & \vdots & \vdots & \ddots & \vdots \\
A_{n 1} & A_{n 2} & A_{n 3} & \cdots & A_{n n}
\end{array}\right] .
$$

\subsection{Full Matrix Focusing Imaging Algorithm}

Imaging algorithm according to the full matrix data is shown schematically in Figure 1. The imaging region below the transducers is discretized into many focus points in alignment. Taking focus point $\boldsymbol{P}$ as an example, the distances between the point $\boldsymbol{P}$ and two elements $\boldsymbol{i}, \boldsymbol{j}$ can be calculated. The amplitude $\boldsymbol{A}_{i j}\left(\boldsymbol{t}_{0}\right)$ in the signal $\boldsymbol{A}_{i j}$ can be determined according to the corresponding travel time $\boldsymbol{t}_{\mathbf{0}}$. The amplitudes from all A-scan signals is superimposed with the same rule. Thus the digital amplitude of the focus point $\boldsymbol{P} I_{\mathrm{P}}(x, z)$ can be calculated according to Eq. (2).

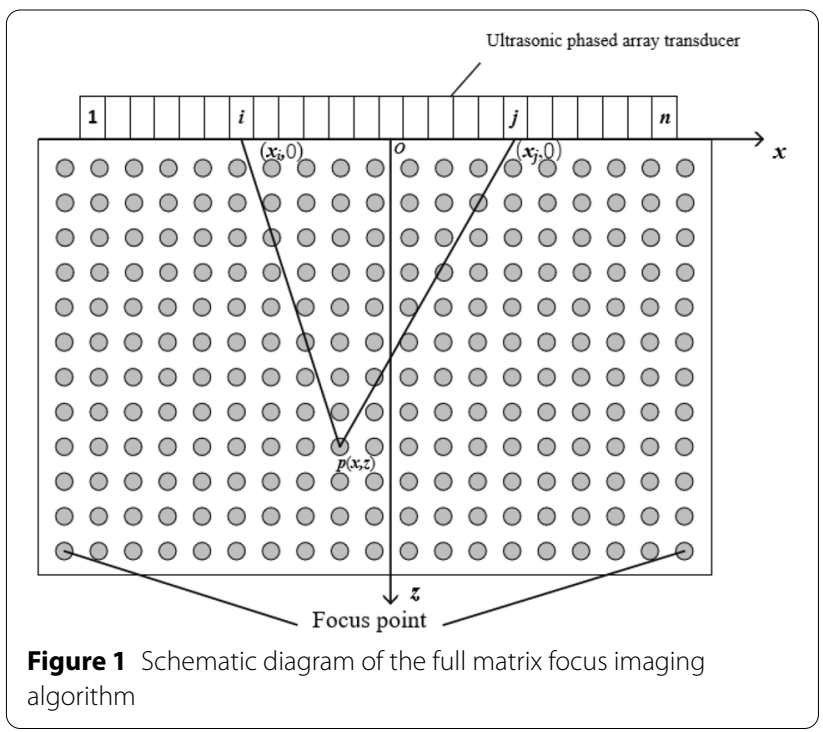

$$
\begin{aligned}
& I_{\mathrm{P}}(x, z)=\sum_{i=1}^{N} \sum_{j=1}^{N} A_{i j}\left(t_{0}\right), \\
& t_{0}=\left(\sqrt{\left(x_{i}-x\right)^{2}+z^{2}}+\sqrt{\left(x_{j}-x\right)^{2}+z^{2}}\right) / c,
\end{aligned}
$$

where $c$ is the wave speed in the material.

\subsection{Triangular Matrix Focusing Imaging Algorithm}

The full-matrix focus imaging algorithm needs all A-scan data acquisition and superposition, so it is a time-consuming method. Considering the reciprocity principle of the multi-channel acoustic system, the transmit and receive channels are interchanged. A good consistency in A-scan amplitudes is maintained. The $\boldsymbol{A}_{i j}$ signal (the $\boldsymbol{i}$ element is transmitted, and the $\boldsymbol{j}$ element is received) should have a good consistency with the $A_{j i}$ signal (the $\boldsymbol{j}$ element is transmitted, and the $\boldsymbol{i}$ element is received). Then, the full matrix data shown in Eq. (1) is a symmetric matrix. It is sufficient that the imaging algorithm only use the upper triangular matrix signal, as shown in Eq. (4). It is called as the triangular matrix focusing imaging algorithm according to Eq. (5).

$$
\left[\begin{array}{ccccc}
A_{11} & A_{12} & A_{13} & \cdots & A_{1 n} \\
A_{21} & A_{22} & A_{23} & \cdots & A_{2 n} \\
A_{31} & A_{32} & A_{33} & \cdots & A_{3 n} \\
\vdots & \vdots & \vdots & \ddots & \vdots \\
A_{n 1} & A_{n 2} & A_{n 3} & \cdots & A_{n n}
\end{array}\right],
$$




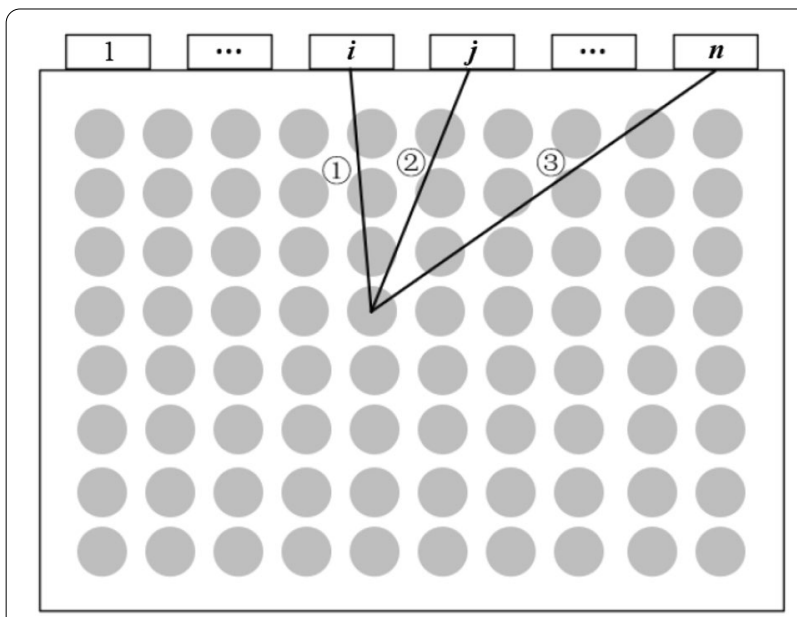

Figure 2 The distance of different receiving elements from the same focus point

$$
I_{\mathrm{P}}(x, z)=\sum_{i=1}^{N} \sum_{j \geq i}^{N} A_{i j}\left(t_{0}\right) .
$$

Comparing Eqs. (1) and (2) to Eqs. (4) and (5), the amount of data acquisition and calculation is reduced from $n \times n$ to $n \times(n+1) / 2$. Then the amount of both data transfer, storage and calculation will be almost reduced by half.

\subsection{Trapezoidal Matrix Focusing Imaging Algorithm}

In order to improve the computational efficiency, the signal energy weights of different channels to the focus point are also considered. Synthetic aperture focusing technique (SAFT) is very useful method. The data on the main diagonal of the matrix is used in SAFT. The transmitting element is same as the receiving element. For a given focus point as shown in Figure 2, the A-scan amplitude is weaken with the distance from the transmitter to the receiver element increasing. Taking path1, path 2 and path 3 in Figure 2 as an example, with the travelled distance increasing from the focus point, the A-scan amplitude of the receiving element $\boldsymbol{i}, \boldsymbol{j}$ and $\boldsymbol{n}$ is weaken gradually.

It is easy to find that the data with larger energy weight are distributed near the diagonal of the triangular matrix. Therefore, the data near to the diagonal of the triangular matrix is reserved, and data far from the diagonal of the triangular matrix is ignored. Then, the trapezoidal matrix focusing imaging algorithm is determined as shown in Eqs. (6) and (7).

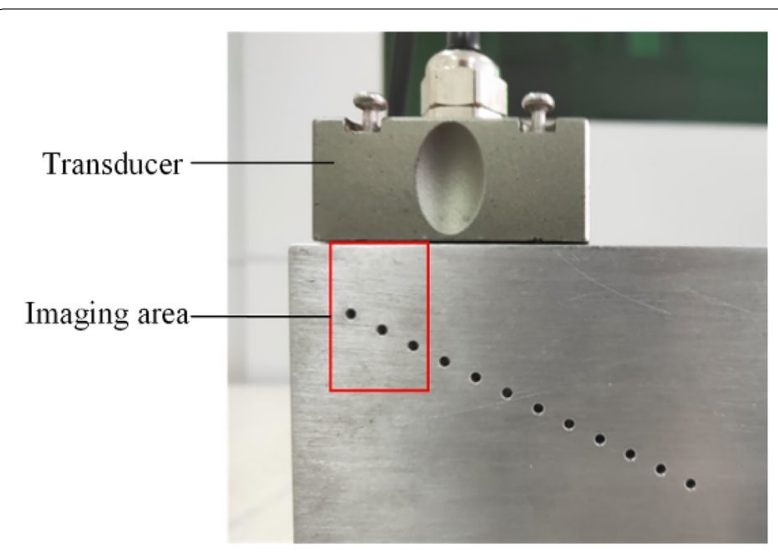

a Side-driller holes defect

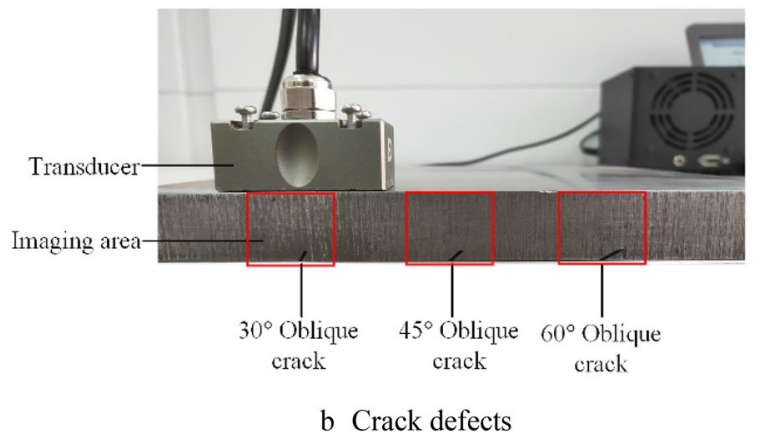

Figure 3 The Experiment Specimens

$$
\begin{aligned}
& {\left[\begin{array}{ccccc}
A_{11} & A_{12} & A_{13} & \cdots & A_{1 n} \\
A_{21} & A_{22} & A_{23} & \cdots & A_{2 n} \\
A_{31} & A_{32} & A_{33} & \cdots & A_{3 n} \\
\vdots & \vdots & \vdots & \ddots & \vdots \\
A_{n 1} & A_{n 2} & A_{n 3} & \cdots & A_{n n}
\end{array}\right],} \\
& I_{\mathrm{P}}(x, z)=\sum_{i=1}^{N} \sum_{i \leq j \leq k}^{N} A_{i j}\left(t_{0}\right),
\end{aligned}
$$

where $i<k<N$.

After obtaining the superposition amplitude of each focusing point, the original image needs to be normalized for the digital display. The 256 colors bar is often used in the ultrasonic NDT field. The superposition amplitudes are normalized from -127 to 128 using Eqs. (8) and (9).

If $I_{\mathrm{P}}(x, z)>0$,

$$
I_{\mathrm{P}}(x, z)=\frac{I_{\mathrm{P}}(x, z)}{I(x, z)_{\max }} \times 128,
$$




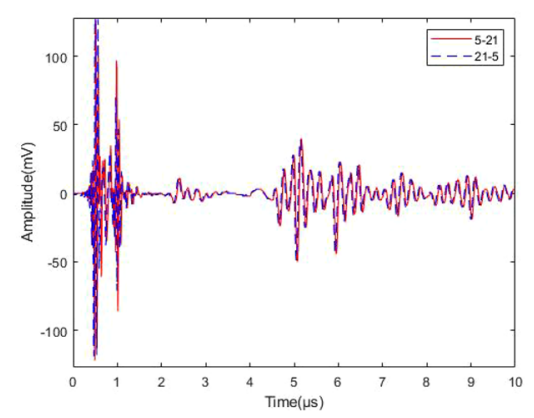

a side-driller holes specimen $A_{5-21}$ and $A_{21-5}$ channel data

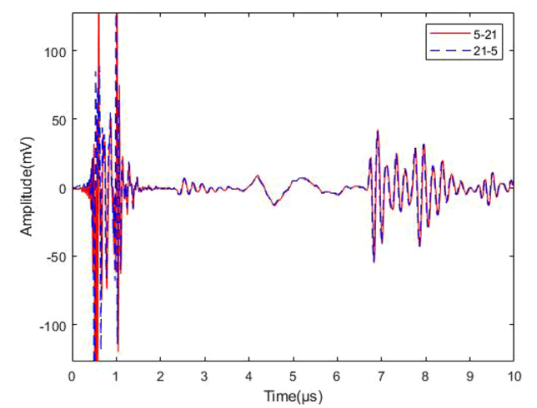

c $45^{\circ}$ crack specimen $A_{5-21}$ and $A_{21-5}$ channel data

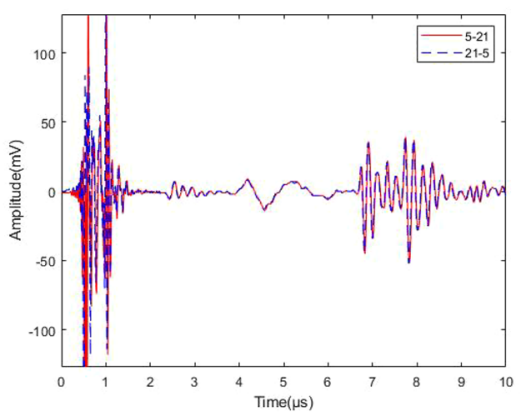

b $30^{\circ}$ crack specimen $A_{5-21}$ and $A_{21-5}$ channel data

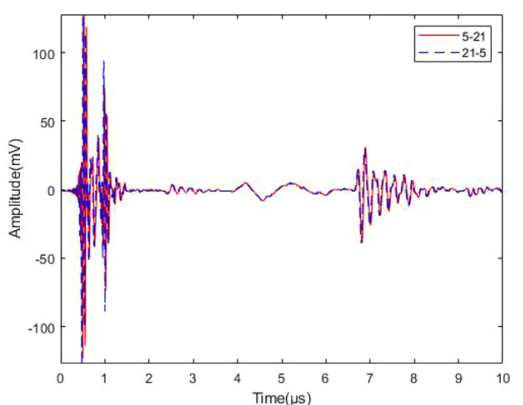

d $60^{\circ}$ crack specimen $A_{5-21}$ and $A_{21-5}$ channel data

Figure 4 Comparison of typical A signals after exchanging transmission and reception channels in specimens

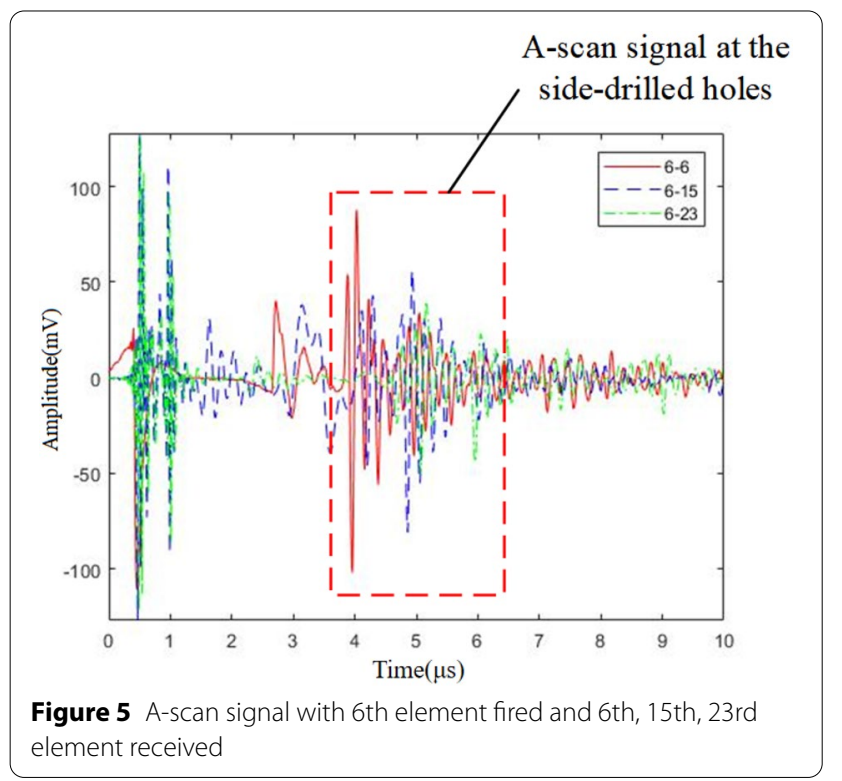

$$
\text { If } \begin{aligned}
I_{\mathrm{P}}(x, z) & \leq 0, \\
I_{\mathrm{P}}(x, z) & =-\frac{I_{\mathrm{P}}(x, z)}{I(x, z)_{\min }} \times 127 .
\end{aligned}
$$

\section{Experimental Verification}

Both symmetric side-drilled holes and asymmetric oblique cracks are used in the experiment. The experiments are analyzed imaging quality and calculation efficiency with different matrix focusing imaging algorithm. The data acquisition and imaging experiments are based on an AOS $64 \times 64$ ultrasonic phased array system and independent-research-and-development imaging software. The sampling frequency of each channel is 100MSPS. A linear array with $5 \mathrm{MHz}$ center frequency and $0.6 \mathrm{~mm}$ element center distance is used in the experiment. Due to the large amount of data collected using the full matrix focus method, only 32 channels and elements are used in the data acquisition.

Symmetric side-driller holes with $1.5 \mathrm{~mm}$ diameter are fabricated in an aluminum alloy specimen, as shown in Figure 3(a). Three asymmetric cracks with different tilt angles of $30^{\circ}, 45^{\circ}$ and $60^{\circ}$ are fabricated at the bottom of the low carbon steel, as shown in Figure 3(b).

\subsection{Comparison of Signal Consistency and Energy Weight} Considering the reciprocity principle of the A-scan signals, the transmitter and receiver channels are interchanged. Some pairs of A-scan signals are selected randomly from the full matrix data, as shown in Figure 4. 


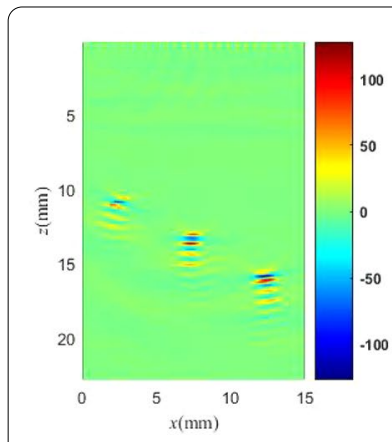

a Full matrix

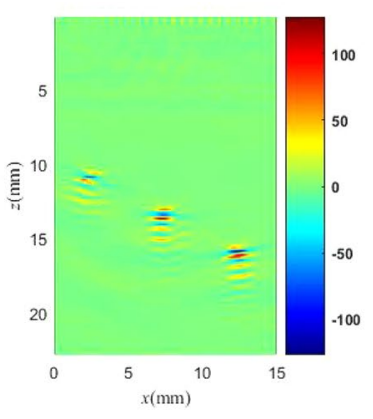

b Triangular matrix

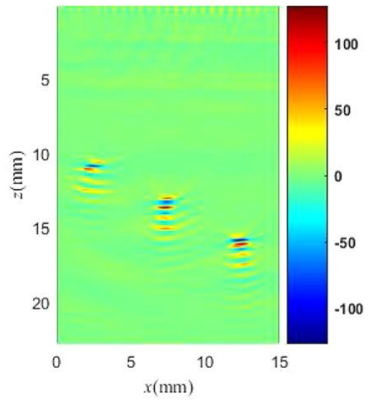

c Trapezoidal matrix $(k=16)$

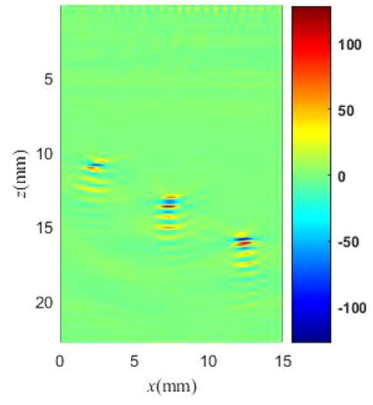

d Trapezoidal matrix $(k=12)$

Figure 6 Comparison of different image methods for side-drilled holes

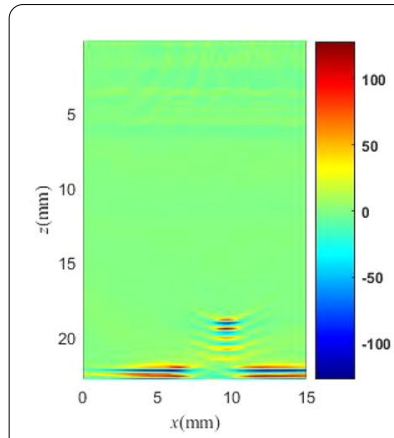

(a) Full matrix

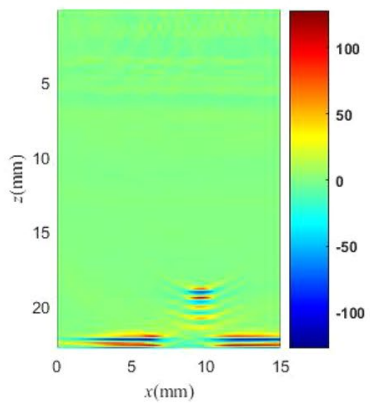

b Triangular matrix

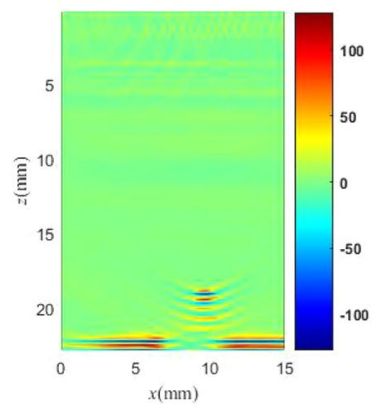

c Trapezoidal matrix $(k=16)$

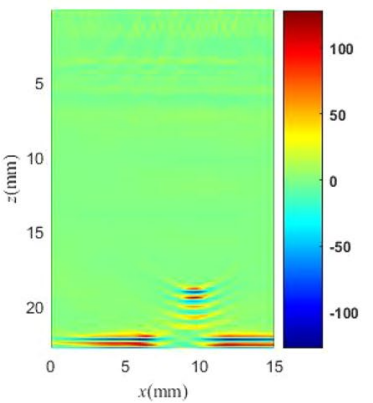

d Trapezoidal matrix $(k=12)$

Figure 7 Comparison of different image methods for $30^{\circ} \mathrm{crack}$

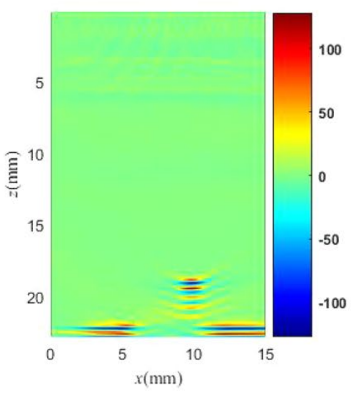

a Full matrix

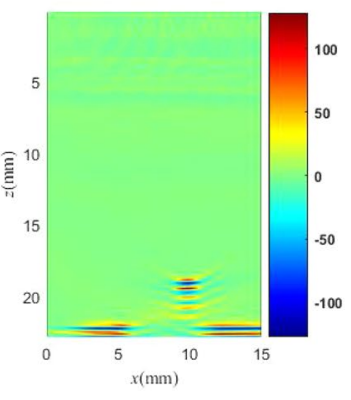

b Triangular matrix

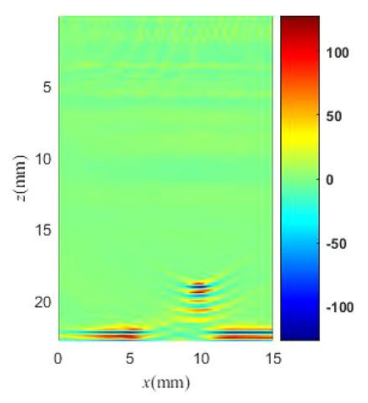

c Trapezoidal matrix $(k=16)$

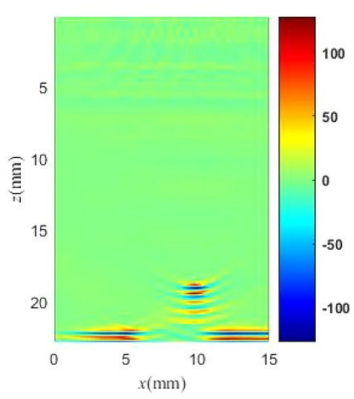

d Trapezoidal matrix $(k=12)$

Figure 8 Comparison of different image methods for $45^{\circ}$ crack

Figure 4(a) shows that the signal of the symmetric sidedrilled holes, and Figure 4(b)-(d) shows the signal of asymmetric oblique cracks. It can be seen from the results that the $\boldsymbol{A}_{5-21}$ signal always keeps good consistency with $\boldsymbol{A}_{21-5}$ signal. Both phase and amplitude of $\boldsymbol{A}_{5-}$ 21 and $A_{21-5}$ are almost same, and signal amplitude in the near field part is slightly different. The rule can also be found in any pair of $\boldsymbol{A}_{i j}$ and $\boldsymbol{A}_{j i}$ channels in the full matrix data. Therefore, the full matrix data is a symmetric matrix.

As shown in Figure 5, A-scan signals are side-drill hole response with 6th element fired and 6th, 15th, 23rd element received. It can be seen that, as the distance from the transmitter to the receiver increasing, the amplitude 


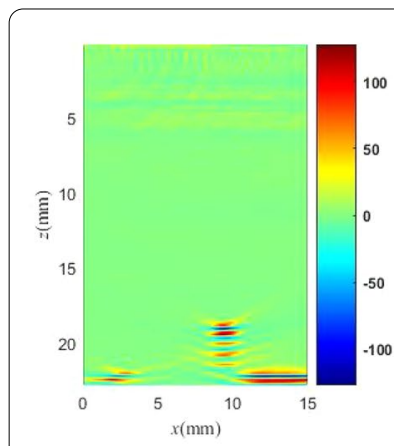

a Full matrix

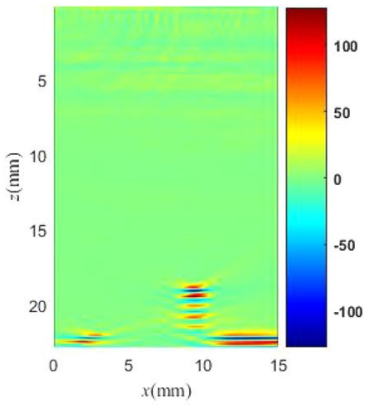

b Triangular matrix

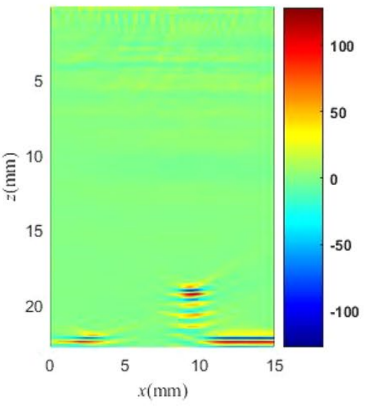

c Trapezoidal matrix $(k=16)$

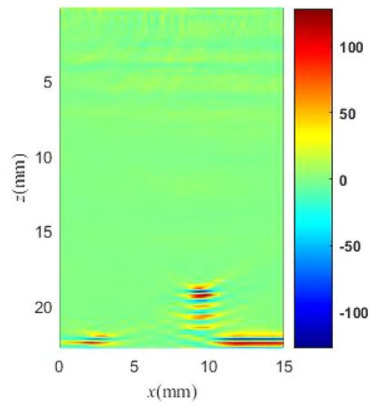

d Trapezoidal matrix $(k=12)$

Figure 9 Comparison of different image methods for $60^{\circ} \mathrm{crack}$

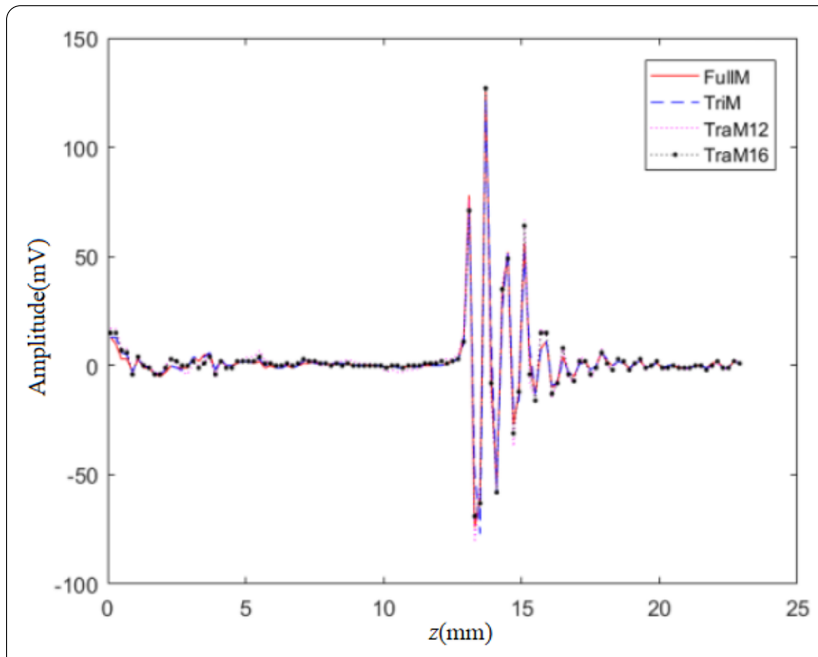

a side-drilled holes

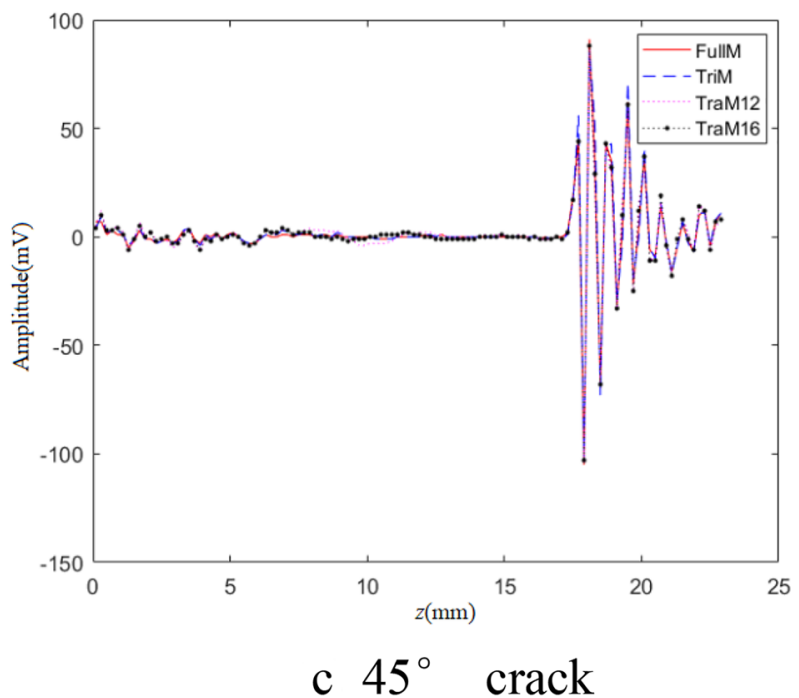

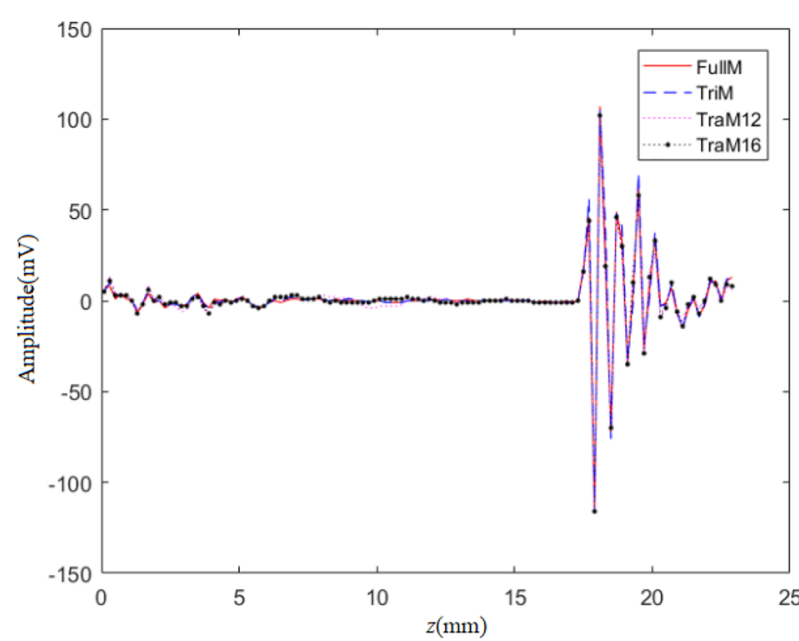

b $30^{\circ}$ crack

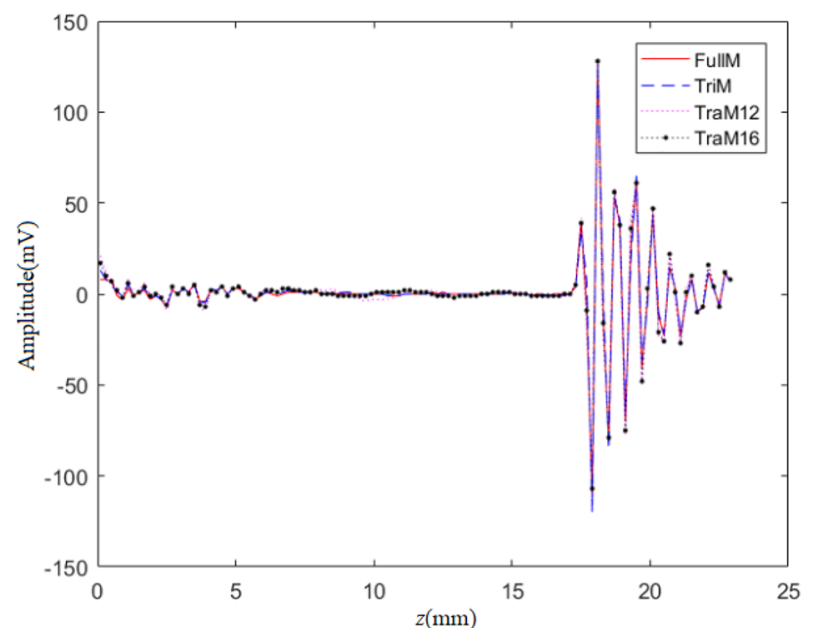

d $60^{\circ}$ crack

Figure 10 Comparison of synthetic A signals with different image methods 

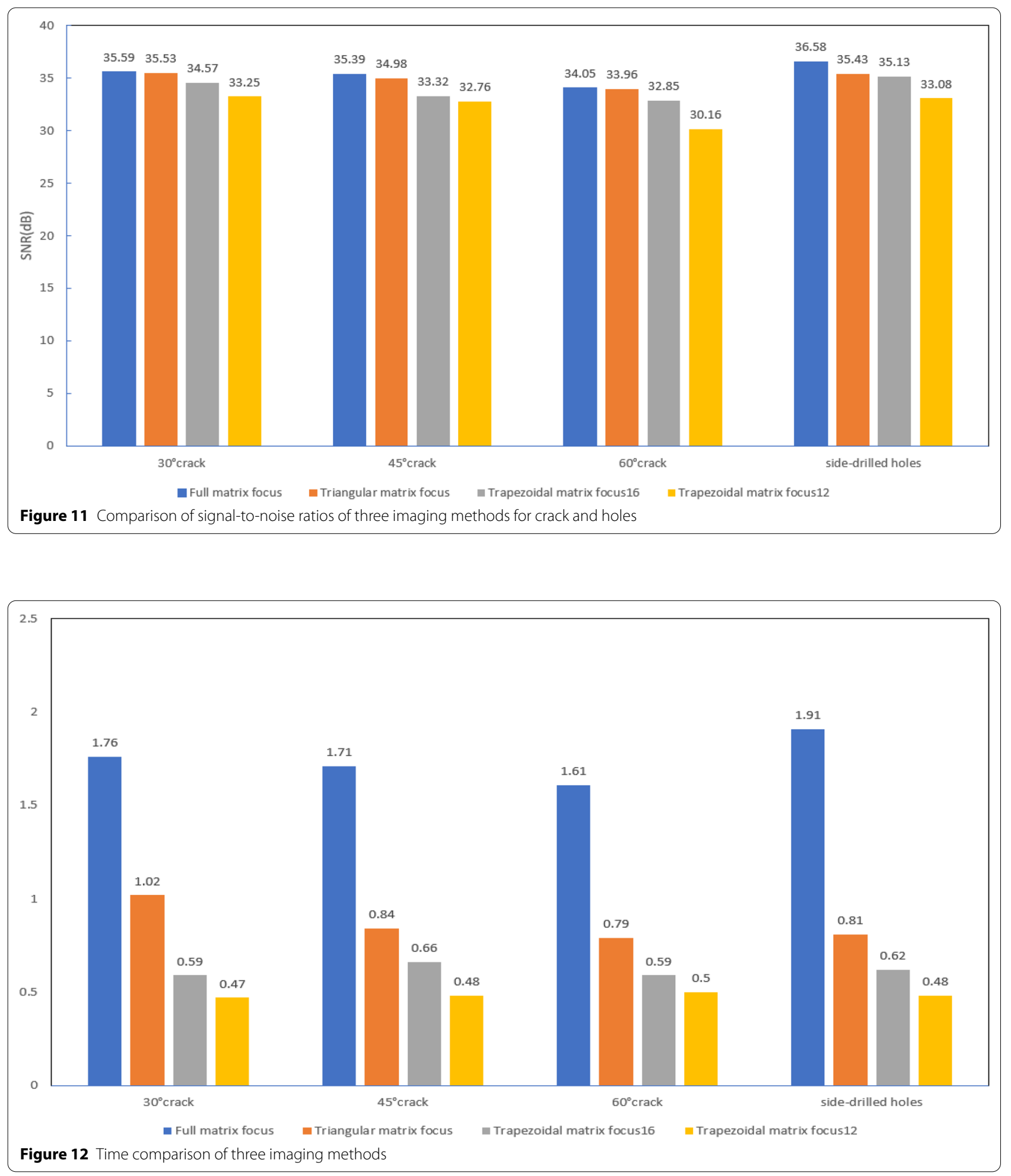
of side-drill hole response is gradually decreased and time delay is increased. The data energy weight is lower as far away from the main diagonal of the matrix.

\subsection{Comparison of Imaging Quality}

Comparison of different matrix focus imaging methods is shown in Figures 6, 7, 8 and 9. Figure 6 shows the different matrix focus imaging of symmetric defects, and Figures 7,8 and 9 show the different matrix focus imaging of asymmetric cracks with oblique angle of $30^{\circ}, 45^{\circ}$ and $60^{\circ}$. It is difficult to identify the difference of the four algorithms in those imaging results observationally. The holes and crack tip signals with different oblique angles can be found clearly. And the crack oblique angle can be evaluated according to the missing position of bottom reflection to the crack tip position as shown in Figures 7, 8 and 9. These patterns can be found in a good consistency with original full matrix focusing method, provided triangular matrix focusing method and provided the trapezoidal matrix focusing method.

In order to analyze consistency of original full matrix focusing method, provided triangular matrix focusing method and provided the trapezoidal matrix focusing method, A-scan signals of side-drilled holes and oblique cracks are shown in Figure 10. It can be seen that amplitude and phase of the synthesized A-scan signals are in good consistency with the three matrix focusing methods.

The signal-to-noise ratio (SNR) of echo signal are calculated separately. The peak value of the signal is regarded as $V_{s}$, and the average amplitude of noise as $V_{n}$. Then the SNR is calculated according to the signal peak value and the average amplitude of noise using Eq. (10).

$$
S N R=20 \times \log _{10}\left(V_{S} / V_{n}\right) .
$$

Echo signal SNR of oblique cracks and side-driller holes are shown in Figure 11 with original full matrix focusing method, provided triangular matrix focusing method and provided the trapezoidal matrix focusing method. Obviously, the SNR of the triangular matrix focusing algorithm is almost the same as that of the full matrix focusing algorithm. And the slightly difference is found in that of the trapezoidal matrix focusing algorithm. As decreasing the number of A-scan data ( $k$ from 16 to 12 ), SNR of the trapezoidal matrix is decreased.

The computational efficiencies of original full matrix focusing method, provided triangular matrix focusing method and provided the trapezoidal matrix focusing method are figured out as shown in Figure 12. B-scan image with $115 \times 150$ pixels is used to compare the imaging time with the three methods. It can be seen that the full matrix focusing is a time-consuming method. The calculation time of the triangular matrix focusing method is cut in half to that of full matrix focusing method. The trapezoidal matrix focusing method spends shorter time as decreasing the number of A-scan data ( $k$ from 16 to 12). Therefore, the computation efficiency can be improved greatly with the help of both provided triangular and trapezoidal matrix algorithms.

\section{Conclusions}

(1) Based on the reciprocity principle, the triangular matrix focusing algorithm is provided to simplify the full matrix focusing method. Furthermore, the trapezoidal matrix focusing algorithm is proposed based on the signal energy weight.

(2) The experimental results of side-drilled holes and oblique cracks show that, three imaging methods keep a great agreement in both B-scan images and synthesized A-scan signals. The signal-to-noise ratio of the triangular matrix is almost same as full matrix focusing algorithm. And the signal-to-noise ratio of the trapezoidal matrix are slightly lower as deceasing the amount of A-scan data.

(3) The triangular and trapezoidal matrix algorithms can achieve the good imaging quality and increase the computation efficiency greatly.

\section{Acknowledgements}

Not applicable.

\section{Authors' contributions}

XZ was in charge of the whole trial; ZM wrote the manuscript; JZ assisted with sampling and laboratory analyses and optimized the manuscript. All authors read and approved the final manuscript.

\section{Authors' Information}

Xinyu Zhao, born in 1979, is currently an associate professor at School of Materials Science and Engineering, Dalian Jiaotong University, China. He received his Ph.D. degree in materials processing and engineering from Harbin Institute of Technology, China, in 2009. His research interests include welding, nondestructive testing and evaluation technology and equipment.

Zemin Ma, born in 1995, is currently a master candidate at School of Materials Science and Engineering, Dalian Jiaotong University, China.

Jiaying Zhang, born in 1988, is currently an associate professor at School of Materials Science and Engineering, Dalian Jiaotong University, China. She received her Ph.D. degree in materials processing and engineering from Harbin Institute of Technology, China, in 2018. Her research interests include non-destructive testing and evaluation technology.

\section{Funding}

Supported by the National Natural Science Foundation of China (Grant No. 51905070).

\section{Competing interests}

The authors declare no competing financial interests. 
Received: 21 December 2020 Revised: 22 September 2021 Accepted: 24 January 2022

Published online: 05 March 2022

\section{References}

[1] G Yang, N Du. Phased array three-dimensional all-focus imaging detection technology. NDT \& E, 2018, 40(5): 64-67.

[2] J P Jiao, S F Yang. Research on phase-weighted vector all-focus ultrasound array imaging method. Acta Acoustics, 2017, 42(4): 485-494.

[3] Y Lin. Ultrasonic phased array full-focus imaging detection. Nondestructive Testing, 2017, 39(5): 57-64

[4] Z Q Zhou, Y Li. Research, application and development of phased array ultrasonic post-processing imaging technology. Chinese Journal of Mechanical Engineering, 2016, 52(6):1-11.

[5] L Lin, P H Yang, D H Zhang. Review of phased array ultrasonic testing for thick wall cast austenitic stainless steel pipeline welds. Journal of Mechanical Engineering, 2012, 48(4): 12-20.

[6] C Li, D Pain, P D Wilcox. Imaging composite material using ultrasonic arrays. NDT \& International, 2013, 53 (16): 8-17.

[7] XY Zhao, T Gang, B X Zhang. Prediction of radiation beam fields from an array transducer with nonparaxial multi-Gaussian beam model. Acta Acustica, 2008, 33(5): 475-480.

[8] XY Zhao, T Gang. Nonparaxial multi-Gaussian beam models and measurement models for phased array transducers. Ultrasonics, 2009, 49(1): $126-130$.

[9] Z Pan, B J Zhang. Quantitative simulation of tilted cracks with ultrasonic phased array all-focus imaging technology. China Test, 2020, 46(4): 36-41.

[10] Y Jing, B Wu. Ultrasonic array full-focus imaging method for anisotropic weld defects. Acta Acoustics, 2019, 44(1): 125-135.

[11] J Q Liu, HY Zhang. Ultrasonic phased array wavenumber imaging algorithm for rail defects. Applied Acoustics, 2018, 37(6): 835-842.

[12] Y Chen, Q Q Ma. Research on fast all-focus ultrasound imaging based on Omega-K algorithm. Chinese Journal of Scientific Instrument, 2018, 39(9): $128-134$.

[13] C Holmes, B W Drinkwater, P D Wilcox. Post-processing of the full matrix of ultrasonic transmit-receive array data for non-destructive evaluation. NDT\&E International, 2005, 38(8): 701-711.

[14] B Wu, J Yang, J P Jiao. Study on ultrasonic multi-mode composite total focusing imaging method for welds defect inspection in small-diameter tube of austenitic stainless steel. Journal of Mechanical Engineering, 2020, 56(14): 9-18.

[15] Z Pan, B J Zhang, H L Wang. Simulation on inclined cracks sizing of using the ultrasonic phased array total focusing imaging technique. China Measurement \& Test, 2020, 46(4): 37-41.

[16] B Wu, J Yang, J P Jiao. Study on ultrasonic array imaging method for aluminum container welds. Journal of Mechanical Engineering, 2020, 56(14): 9-18.

[17] J G Wang, H Yao, H J Luo. Study on ultrasonic array imaging method for aluminum container welds. Experimental Mechanics, 2018, 33(4): 517-524.

[18] XW Shen. Full focus imaging of sparse array ultrasonic phased array based on particle swarm optimization. Applied Acoustics, 2020, 39(3): 354-359.

[19] S A Yang. Design of ultrasonic phased array real-time full focus system based on FPGA. Wuhan: University of Chinese Academy of Sciences (Wuhan Institute of physics and mathematics, Chinese Academy of Sciences), 2020

[20] P J Chen. Debonding detection of composite shell engine based on phased array ultrasound. Taiyuan: Zhongbei University, 2021.

[21] J Zhang, M R Yang. Comparative analysis of ultrasonic phased array full focus imaging algorithms. Acoustic technology, 2021, 40(1): 71-76.

[22] Y Chen, Q Q Mao, G Chen. Research on fast full focus ultrasound imaging based on omega-k algorithm. Journal of Instrumentation, 2018, 39(9): 128-134.

[23] H Q Wu. Acquisition method of coded ultrasonic sparse data and plate weld defect imaging based on FPGA. Zhenjiang: Jiangsu University, 2020.

[24] M L Zhao. Research on defect detection of double-layer structure based on full matrix phase transfer algorithm. Chengdu: Southwest Jiaotong University, 2020.
[25] L C Jia, S L Chen, Z L Bai. Calibration model and acceleration algorithm of ultrasonic full focus imaging. Journal of instrumentation, 2017, 38(7): 1589-1596.

[26] C Ran. Research on fast ultrasonic full focus imaging based on FPGA. Chengdu: Southwest Jiaotong University, 2017.

[27] H R Jin, J Chen. An efficient wavenumber algorithm towards real-time ultrasonic full-matrix imaging of multi-layered medium. Mechanical Systems and Signal Processing, 2021: 149.

[28] H Zhang, J Zheng, B Bai. Optimal design of sparse array for ultrasonic total focusing method by binary particle swarm optimization. IEEE Access, 2020, 8: 111945-111953.

[29] H W Hu, J Du. Ultrasonic phased array sparse-TFM imaging based on sparse array optimization and new edge-directed interpolation. Sensors, 2018, 18(6): 1830-1845.

[30] HW Hu, J Du, N Xu. Ultrasonic sparse-TFM imaging for a two-layer medium using genetic algorithm optimization and effective aperture correction. NDT \& E International, 2017, 90: 24-32.

\section{Submit your manuscript to a SpringerOpen ${ }^{\circ}$ journal and benefit from:}

- Convenient online submission

- Rigorous peer review

- Open access: articles freely available online

- High visibility within the field

- Retaining the copyright to your article

Submit your next manuscript at springeropen.com 